\title{
La figura femminile nella Raffaella di Alessandro Piccolomini e nelle sue traduzioni francesi
}

\author{
Daniela Costa
}

La Raffaella, o Dialogo della bella creanza delle donne ${ }^{1}$ di Alessandro Piccolomini, pubblicato nel 1539 sotto lo pseudonimo accademico di Stordito Intronato, ${ }^{2}$ ebbe in Francia diverse traduzioni: l'individuazione delle modifiche che le figure femminili del dialogo italiano subirono lungo il percorso costituisce il tema del presente contributo, il quale intende fornire, in questa sede, solamente alcuni accenni preliminari.

Il dialogo del Piccolomini, che è stato definito una "scena di commedia dilatata con materiale didascalico" (Di Benedetto 25) ed un'opera dalla "fisionomia intenzionalmente anomala, in bilico tra procedura didattica [...] e spirito di parodia" (Baldi, "La Raffaella" 666) presenta due personaggi indimenticabili: ${ }^{3}$ la vecchia mezzana Raffaella, che ostenta nobili natali, e la giovane sposa Margarita, appartenente all'alta società senese, spesso costretta alla solitudine durante le lunghe assenze del marito in viaggio per affari. Raffaella ha tutti i tratti letterari della mezzana: "entremetteuse bigote" e "corruptrice eloquente" (Lazard 207), si finge devota e pietosa per dare alle proprie parole maggiore credibilita, e la sua abile retorica rivela una fine conoscenza della psicologia maschile e femminile. Il dialogo è vivace, ricco di espressioni tratte dal parlato senese, intessuto di riferimenti alla vita quotidiana del tempo, a personaggi reali evocati per metterne in ridicolo i difetti o compatime la sorte. Le due donne sono personaggi vivi, ed i tratti del loro carattere rimangono impressi nella memoria del lettore: Raffaella è infatti una mezzana che alle caratteristiche fissate dalla tradizione letteraria aggiunge tratti originali, di grande effetto scenico; Margarita segue docile il ragionamento di Raffaella e si trasforma da giovane ingenua, pettegola ed un po' sciocca in amante appassionata. La conversazione offre a Raffaella l'occasione di mostrare la propria abilità dialettica nel riuscito tentativo di convincere la giovane Margarita ad accettare le profferte amorose del pretendente Aspasio, probabilmente mandante della mezzana. Raffaella si presenta alla compassione di Margarita come una gentil-donna ormai anziana, caduta in povertà ed amica di famiglia, già stimata dalla madre della giovane sposa; la mezzana ostenta la sua assiduità alle funzioni religiose, insistendo sul desiderio di rimediare ai propri peccati, presentandosi in tal modo come un possibile 
"directeur de conscience" (Piéjus, "Vénus bifrons" 127) della giovane Margarita. Questa devozione pero si rivela ben presto per quello che è, abile atto ipocrita, quando il discorso di Raffaella rovescia la nozione stessa di peccato, sfiorando il blasfemo. La mezzana dice infatti di provare rimorso per cio che non ha fatto, per la gioventù che ha lasciato trascorrere senza coglierne i frutti, e confessa che la bellezza di Margarita, ponendole sotto gli occhi la propria vecchiaia, la gettá nella disperazione. I discorsi di Raffaella rivelano la sua intelligenza ed abilità retorica: nel rovesciamento di valori da lei operato, la "vera carità" risulta infatti essere proprio la sua attività di consigliera delle giovani, in qualità di donna alla quale la "pratica del mondo" (Dialogo 472) ha insegnato a distinguere il male dal bene e ad evitare gli errori. La logica di Raffaella è stringente: l'uomo è nato peccatore, e "qualche erroruzzo in gioventù" impedisce di cadere in tarda età nella "desperazione" (473). L'obiettivo dichiarato dalla mezzana è quindi quello di insegnare a Margarita il modo di gustare i piaceri della giovinezza garantendosi una convivenza pacifica con il marito e conservando la propria reputazione fra i concittadini. ${ }^{4}$ Il discorso si fa poi precettistico: Raffaella educa Margarita all'eleganza, le insegna ad avere gusto nella scelta delle stoffe, nel taglio delle vesti e nell'abbinamento dei colori. Tuttavia la qualità necessaria per valorizzare l'abito è la "portatura" (484): i consigli di Raffaella sull'abbigliamento presuppongono un "garbo pien di giudizio" (483), un portamento discreto che consiste in una perfetta misura di gesti, vera essenza di una naturale eleganza. Al fine di illustrare gli atteggiamenti da evitare, Raffaella traccia i ritratti, confinanti con la caricatura, di donne senesi del tempo, ben conosciute dalle due interlocutrici, offrendo a Margarita l'occasione di sfoggiare il suo gusto del pettegolezzo. La conclusione che ne segue è che "molto giova lo ingegnarsi che in tutte le cose non si esca de la via del mezzo e fuggire la affettazion più che si può" (498): sulla scia della sprezzatura castiglionesca, l'aurea mediocritas diventa la regola generale del comportamento. L'abilità retorica di Raffaclla ha serbato per ultimo l'argomento decisivo e più importante, l'amore, esaltato quale componente fondamentale della vita umana ed in particolare della giovinezza, che senza di esso perde ogni valore e significato. Raffaella insegna alla giovane allieva a scegliere con cura un amante degno di fiducia, per poter condurre una relazione amorosa felice e duratura, offrendo nel suo discorso un'indimenticabile serie di ritratti maschili, dai "giovani vantatori e parabolani" (514) al vecchio "malissima lingua ed invidioso" (516). Gli aggettivi si susseguono in un ritmo incalzante e vorticoso, e rivelano il vuoto, l'inconsistenza morale che si celano dietro tali personaggi. Il catalogo degli uomini da evitare è anch'esso funzionale al disegno retorico di Raffaella: esso infatti serve a delineare il ritratto dell'amante ideale, che la mezzana farà abilmente coincidere con quello del pretendente messer Aspasio. Il dialogo si chiude sul commiato delle due donne, e Margarita si 
affida all'opera mediatrice di Raffaella, promettendole una generosa ricompensa.

Raffaella risulta essere la vera protagonista della conversazione, maestra di eleganza femminile ma anche di eloquenza: il passo, verso la fine del dialogo, in cui con slanci bembistici Raffaella fa un commosso elogio dell'amore, ne è una riprova. Di fronte al ritratto indimenticabile di Raffaella, Margarita appare una figura insignificante, facilmente manovrabile, di un'ingenuità dovuta non tanto ad un innato candore quanto piuttosto ad un'ottusa ignoranza causata da una scarsa esperienza della vita in societa. Margarita rappresenta un interlocutore "necessario perché quell'iniziazione si attui, non perché i suoi interventi collaborino a una dimostrazione conclusiva" (Borsellino 26): le battute della giovane sposa consistono per lo più in domande aventi la funzione di far procedere il discorso ed in allusioni pettegole ad avvenimenti ed a personaggi senesi, al fine di inserire i precetti di Raffaella nella realtà quotidiana, riducendone la portata teorica per comprenderli ed assimilarli.

I personaggi conservano un profilo psicologico simile anche nella prima traduzione francese della Raffaella, pubblicata nel 1572 sotto il titolo di Instruction pour les jeunes dames e firmata M.D.R. ${ }^{5}$ Il dialogo è una traduzione letterale dell'operetta del Piccolomini ma con una leggera modifica nel finale: dietro la mère non compare infatti l'ombra di un mandatario, ed il suo discorso appare quindi disinteressato (a differenza di Raffaella la mère non accenna infatti alla sua povertà, né ad eventuali ricompense) e volto ad offrire una guida ai piaceri dell'amore, come prospettato nella lettera di dedica. Se la figura della fille ricalca, nel corso del dialogo, quella di Margarita, essa assume però nuove sfumature alla luce della lettera di dedica "Aux jeunes dames" dell'Instruction, nella quale M.D.R. dichiara di trascrivere una conversazione realmente avvenuta con una parente. L'autrice si identifica con la fille interlocutrice del dialogo: secondo la finzione letteraria presentata nella lettera di dedica, l'esistenza stessa dell'opera presuppone quindi una fille ormai pienamente consapevole di sé, che ha attuato i consigli della mere, scoprendo le gioie dell'amore. Con l'aggiunta della lettera di dedica, la fille muta fisionomia e supera la passività di Margarita per assumere un ruolo attivo, offrendosi di "instruire" le sue coetanee, ancora ignare dell'amore. Inoltre, M.D.R. suggerisce il motivo della presenza degli appellativi di mère e fille, attribuendo a quest'ultima l'iniziativa. Dalla lettera di dedica apprendiamo infatti che la qualifica di mère è posteriore alla conversazione avvenuta fra le due donne, in segno della riconoscenza della giovane verso colei che le dato vita una seconda volta, indicandole la strada per la realizzazione di un amore felice. In questo modo M.D.R. sottolinea l'importanza degli appellativi mère e fille, che nel dialogo del Piccolomini attestavano semplicemente l'esistenza di legami affettivi fra le due donne anteriori alla conversazione rappresentata nell'opera, ed offre al personaggio della fille la possibilità di trasformarsi in una consapevole M.D.R., dal carattere più deciso e volto all'azione. La lettera 
di dedica, saldamente costruita dal punto di vista retorico, utilizza la finzione narrativa della veridicità del dialogo e dei personaggi per presentare un'opera divertente e divertita, che mette in risalto gli aspetti maliziosi e provocatori dell'operetta del Piccolomini, aprendo così la strada alla lettura secentesca della Raffaella come testo erotico, vicino alle opere dell'Aretino. ${ }^{6}$

L'impostazione didattica e normativa della Raffaella sono riprese dalla seconda traduzione francese del dialogo del Piccolomini, il Notable discours del 1577. Il traduttore, che nella lettera di dedica "A belle, honeste et vertueuse Damoyselle C.D.B." (f. $A^{2}$ r) si firma T.D.C., muta il nome dei personaggi italiani in quelli di Béatrice e Florimonde. Il testo di T.D.C. sarà ripreso da François d'Amboise ${ }^{7}$ nei Dialogues et devis des damoiselles, pubblicati a Parigi nel 1581 sotto lo pseudonimo di Thierri de Timophile e dedicati a Anne d'Atri, colta e bella dama italiana del seguito di Caterina de' Medici, e novella sposa del ricco finanziere fiorentino Ludovico Adiaceto (cfr. Picot). Il testo di T.D.C. viene suddiviso in due dialoghi, corrispondenti ad altrettante giornate di conversazione fra le due interlocutrici, subendo modifiche in apertura e nel finale. Nel Notable discours di T.D.C. e nei Dialogues di François d'Amboise, Béatrice è una colta e saggia dama che ha vissuto a corte, e che intrattiene la giovane Florimonde con una conversazione piacevole, che modera gli accenti licenziosi del dialogo italiano e della traduzione di M.D.R.. François d'Amboise e T.D.C. non accennano infatti al motivo della disperazione causata dalla bellezza della giovane interlocutrice che induce Raffaella e la mère al rimpianto della giovinezza ormai trascorsa: il tono della conversazione resta sempre lontano dagli eccessi, mantenendosi in una costante mediocritas di contenuti e di forme. Il dialogo si presenta come la continuazione di una discussione iniziata tempo prima, in occasione del matrimonio di una cugina di Béatrice in una ristretta cerchia di dame dalla quale gli uomini sono esclusi, all'ombra di un grande faggio e vicino ad una fontana, in una cornice idillica molto simile a quella del Decameron del Boccaccio o degli Asalani del Bembo. Florimonde ricorda a Béatrice la promessa che costei fece, di approfondire in altro tempo e luogo la questione dei modi convenevoli ad una damoiselle per inserirsi nella società evitando di cadere nelle trappole tese dall'astuzia maschile. L'opera si pone quindi fin dall'inizio sotto il segno della conversazione, alla quale Florimonde, a differenza di Margarita, partecipa attivamente. Una chiave di lettura degli adattamenti di T.D.C. e di François d'Amboise è fornita dalla lettera di dedica dei Dialogues, dove l'autore dichiara di rappresentare "deux dames bien advisees, et des mieux disantes de leur aage" (f. $\mathrm{A}^{2}$ r): l'opera si presenta così quale modello di conversazione mondana e femminile. Funzionali allo scopo di elevare il tono del discorso sono alcune modifiche apportate da T.D.C. e riprese da François d'Amboise al testo del Piccolomini. Laddove Raffaella alludeva a personaggi della vita senese conosciuti da entrambe le donne, Béatrice fa 
riferimento a conoscenze non condivise da Florimonde, ridimensionando in tal modo la "naturale vocazione pettegola" (Di Benedetto 25) delle due interlocutrici, per lasciare spazio ad una conversazione sempre misurata ed elegante, a volte maliziosa, mai volgare, esempio di un linguaggio femminile colto e raffinato.

Nella Raffaella gli interventi di Margarita avevano uno scopo puramente funzionale al procedere dell'azione dialogica: Margarita poneva infatti domande, dubbi e curiosità riguardanti i precetti di eleganza e di condotta forniti da Raffaella, offrendole così l'occasione di approfondire alcuni aspetti didattici e normativi del suo discorso. Mentre nell'Instruction gli interventi della fille seguono da vicino quelli di Margarita e ne conservano la stessa funzione strutturale, nel Notable discours e nei Dialogues le riflessioni di Florimonde assumono maggiore rilievo ed autonomia. Essi infatti oltre ad essere strettamente funzionali allo svolgimento della conversazione, sono volti a mettere Florimonde sullo stesso piano culturale di Béatrice, attraverso la dimostrazione delle sue qualità intellettuali in lunghi monologhi dal tono filosofico su temi quali la gloria, la vecchiaia, la morte, che divengono per la giovane interlocutrice meri pretesti per sfoggiare la propria cultura ed abilità retorica. Florimonde si rivela così essere insieme la destinataria e la protagonista dei discorsi normativi di Béatrice: la giovane interlocutrice infatti dimostra continuamente, attraverso i suoi interventi, di possedere già quelle qualità intellettuali e morali (oltre a quelle fisiche) che sono attribuite alla perfetta damoiselle. D'altra parte la stessa Béatrice riconosce che il ritratto da lei tracciato della damoiselle ideale trova una corrispondenza reale nella figura di Florimonde, che unisce alla giovane età una saggezza senile, ed alla bellezza fisica quella spirituale. ${ }^{8}$ Riprendendo il testo del Notable discours, d'Amboise spezza i lunghi monologhi di Béatrice, attribuendone alcune frasi a Florimonde. Autore di teatro, d'Amboise in questo modo intendeva certamente dare alla conversazione un andamento più vivo e più svelto, che ricalcasse quello della battuta di commedia: lo stesso brano di apertura del dialogo di T.D.C. era stato sostituito da d'Amboise con una vera e propria scena teatrale. Una delle conseguenze immediate di tale intervento operato da François d'Amboise sul testo di T.D.C. consiste in un'esaltazione dei caratteri della figura di Florimonde, che assume un maggior rilievo: i suoi interventi risultano ora più frequenti, e costituiscono una conferma della sua cultura e saggezza. Nei Dialogues Florimonde partecipa ancora più attivamente alla conversazione, narrando una novella, dopo averne ascoltata una da Béatrice, e nel finale si fa la portavoce di un elogio appassionato dell'amore coniugale, nel quale è forse da vedere un omaggio alla dedicataria, da poco convolata a nozze, della quale Florimonde costituisce il fedele "portrait", come l'autore dichiara nella lettera di dedica, mentre dietro le reticenze ironiche di Béatrice si nasconde la parola maliziosa e galante dell'autore. ${ }^{9}$ 
Questo nuovo profilo psicologico dei personaggi, ben differente da quello tracciato dalla Raffaella e dall'Instruction, è strettamente correlato al nuovo significato dell'opera. Il dialogo rappresentato nel Notable discours e nei Dialogues si fa trattato, mettendo in scena due colte dame in una conversazione dal tono mondano, modellata su quella di corte del Cortegiano; in effetti, T.D.C. e d'Amboise riprendono quasi alla lettera numerosi brani dell' opera del Castiglione, ed in particolare dal terzo libro, volto a "formare" la perfetta "donna di palazzo". Inoltre, proprio la corte è il punto di riferimento costante dei discorsi di Béatrice, il luogo naturale di un sapere che "comincia dal conversare e finisce nel conversare" (Quondam 1, 30): per giustificare il tono filosofico che il discorso sull'amore sta per assumere, l'anziana dama dice infatti di ripetere $\mathrm{i}$ discorsi uditi un tempo alla corte da dotti gentiluomini, mettendo in atto una captatio benevolentiae al lettore, con un estremo sfoggio di quella "modestie" che precedentemente Béatrice aveva proposto come ornamento delle qualità fisiche ed intellettuali della perfetta damoiselle. Nella bella, giovane e colta Florimonde le dedicatarie del Notable discours e dei Dialogues, C.D.B. e Anne d'Atri, potevano facilmente riconoscersi, così come tutte le colte damoiselles dell'alta società che in Florimonde vedevano quella somiglianza sufficiente e necessaria per farne un modello. Dedicati ad illustri dame dell'alta società lionese e parigina delle quali ricercavano il favore, i testi di T.D.C. e di François d'Amboise si inserivano in un contesto di relazioni mondane: essi non potevano quindi non presentare personaggi radicalmente differenti da quelli della Raffaella e dell'Instruction. Opere di intrattenimento, volte a catturare l'attenzione di un pubblico femminile colto e desideroso di novità, il Notable discours ed i Dialogues intendevano offrire, conformemente al precetto oraziano del delectando docere, un'opera divertente ed utile, in grado di fornire consigli sui modi di vivere in società. Le annotazioni marginali ai testi, volte a segnalare concetti di rilievo ed a riassumerli in sentenze, confermano questo aspetto normativo: non solo divertissement mondano, il Notable discours e soprattutto i Dialogues (che aggiungono una Table degli argomenti trattati) potevano farsi manuale di comportamento e di conversazione, smorzando il tono precettistico con novelle, sentenze ed aneddoti. Mentre l'Instruction conservava il carattere ludico dell'originale italiano e ne riproduceva il tono malizioso e divertito di provocazione ed invito all'adulterio, sotto la finzione di una solidarietà femminile, gli adattamenti di T.D.C. e d'Amboise sembrano porsi in una relazione ben diversa con il pubblico, pur sempre femminile, al quale si rivolgono. La trasformazione del dialogo del Piccolomini nel Notable discours e nei successivi Dialogues di François d'Amboise passa quindi in primo luogo attraverso una radicale modificazione del profilo psicologico dei personaggi, i quali, per poter essere presi sul serio ed adempiere così alla loro funzione di modelli di comportamento e di retorica, devono presentare una certa omogeneità con il pubblico 
al quale si rivolgono. Così, sul modello aretiniano prevale ora quello castiglionesco, e da dialogo ironico e provocatorio l'opera del Piccolomini si trasforma fino a diventare, come abbiamo già detto altrove, un vero e proprio trattato mondano rivolto alle giovani damoiselles dell'alta società francese, sotto la forma di una colta e piacevole conversazione fra due dame sui modi per raggiungere la felicità amorosa.

\section{Universitd di Torino}

\section{NOTE}

1 L'edizione più recente è quella curata da Di Benedetto, Prose. Sulle precedenti edizioni della Raffaella cfr. 25-29 e 459-62. Sul dialogo del Piccolomini si vedano in particolare Pancrazi 99107; Valeri 95-105; Di Benedetto, Stile 90-96 e 108-10; Scognamiglio, "ll dialogo"; Piéjus; e Cope.

2 Su Alessandro Piccolomini si vedano in particolare la monografia di Cerreta; Scrivano 13-50; Celse 7-76. Si vedano inoltre gli studi recenti di Andrea Baldi, "Sperimentazione", "Alessandro Piccolomini e l'Accademia", e "Alessandro Piccolomini, tra impegno".

3 Una fine analisi del ruolo dei personaggi nella struttura del dialogo si ritrova nel denso contributo di Piéjus, "Vénus bifrons", al quale rimandiamo anche per un 'interpretazione del significato dei nomi di Raffaella, Margarita ed Aspasio, il mandatario della mezzana (127).

4 Le fonti dell'operetta del Piccolomini sono moltissime: fra queste, ricordiamo l'Ars amandi di Ovidio, l'Ecatonfilea di Leon Battista Alberti, ed i Ragionamenti dell'Aretino, pubblicati pochi anni prima della Raffaella, oltre alla commedia latina ed ai trattati medievali di igiene e cosmesi.

5 Il testo di M.D.R. fu riedito nel 1573 e nel 1597. L'opera è generalmente attribuita a Marie de Romieu o a Madeleine Des Roches: Scognamiglio (21-6) identifica l'autrice con la prima.

6 Per uno sviluppo dell'argomento, ci permettiamo di rinviare al nostro contributo (Costa).

7 Per un 'interpretazione dei nomi di Béatrice e Florimonde, cfr. Ughetti 194.

8 ll personaggio di Florimonde sembra presentare le caratteristiche tradizionali del puer-senex (Curtius 122-25).

9 D'Amboise suddivide il testo del Notable discours in due parti, corrispondenti a due giomate di conversazione: tale operazione gli permette di inserire alla fine della seconda giomata alcune novelle, con il pretesto che è ancora giomo e vi è ancora tempo per la piacevole conversazione, secondo la topica della conclusione (Curtius 110-13).

\section{OPERE CITATE}

Baldi, Andrea. "Alessandro Piccolomini e l'Accademia degli Infiammati: note in margine ad un dibattito linguistico". Italian Culture 9 (1991): 115-27.

53-65. "Alessandro Picc Jlomini, tra impegno filogino e parodia". Italian Culture 10(1992): "La Raffaella di Alessandro Piccolomini: il trattato vòlto in gioco". Passare il tempo. La letteratura del gioco e dell intrattenimento dal XII al XVI secolo. Atti del Convegno di Pienza 10-14 settembre 1991. Ed. Enrico Malato e Michelangelo Picone. Roma: Salemo Editrice 1993. 665-77.

—. "Sperimentazione letteraria e impegno divulgativo nell'opera di Alessandro Piccolomini”. DAl 53 (1993): 2394A. U of Califomia, Los Angeles.

Borsellino, Nino. Introduzione ad Antonio Vignali. La Cazzaria. Ed. Pasquale Stoppelli. Roma: Edizioni dell'Elefante, 1984. 7-27. 
Celse, Mireille. "Alessandro Piccolomini, l'homme du ralliement". Les écrivains et le pouvoir en Italie à l'époque de la Renaissance. Ed. André Rochon et al. 2 vols. Première série. Paris: Université de la Sorbonne Nouvelle, 1973. 7-76.

Cerreta, Florindo. Alessandro Piccolomini. Letterato e filosofo senese del Cinquecento. Siena: Accademia degli Intronati, 1960.

Cope, Jackson I. Secret Sharers in Italian Comedy from Machiavelli to Goldoni. Durham, North Carolina, and London: Duke UP, 1996.

Costa, Daniela. "La réception française de la Raffaella d'Alessandro Piccolomini. Versions 'urbaines' et lectures “érotiques"'. Nouvelle Revue du Seizième Siècle 14.2 (1996): 237-46.

Curtius, Emst Robert. La littérature européenne et le Moyen âge latin. Trans. Jean Bréjoux. Paris: P.U.F., 1956.

D'Amboise, François. Dialogues et devis des damoiselles, pour les rendre vertueuses et bienheureuses en la vraye et parfaicte amitié. Contenans plusieurs bons enseignemens, tres-utiles et profitables à toutes personnes: enrichis de quelques histoires facetieuses, et discours de la nature d'amour, pour bien et honnestement se gouverner en toutes compagnies. Paris: Vincent Norment, 1581. Parigi, Bibliothèque Nationale [Rés. Z2449].

Di Benedetto, Arnaldo. Stile e linguaggio. Roma: Bonacci, 1974.

Guazzo, Stefano. La civil conversazione. Ed. Amedeo Quondam. 2 voll. Modena: Panini, 1993.

Lazard, Madeleine. Images littéraires de le femme à la Renaissance. Paris: P.U.F., 1985.

Notable discours, en forme de dialogue, touchant la vraye et parfaicte amitié, duquel toutes personnes, et principalement les dames, peuvent tirer instruction utile et profitable, d'autant que par la plaisante lecture d'iceluy elles y sont deuement informees du moyen qu'il faut tenir pour bien et honnestement se gouverner en amour. Lyon: Benoist Rigaud, 1577. Parigi, Bibliothèque Nationale [Rés. p. Y² 154].

Pancrazi, Pietro. Nel giardino di Candido. Firenze: Le Monnier, 1950.

Piccolomini, Alessandro. Dialogo della bella creanza delle donne. Prose di Giovanni Della Casa ed altri trattatisti cinquecenteschi di comportamento. Ed. Amaldo Di Benedetto. Torino: Utet, 1991.

Picot, Emile. "Les Italiens en France au XVIe siècle". Bulletin Italien 3 (1903): 21-22.

Piéjus, Marie-Françoise. "L'Orazione in lode delle donne di Alessandro Piccolomini". Giornale Storico della Letteratura Italiana 170 (1993): 524-50.

. "Vénus bifrons: le double idéal de la femme dans La Raffaella d'Alessandro

Piccolomini". Images de la femme dans la littérature française de la Renaissance. Ed. André Rochon et al. Paris: Université de la Sorbonne Nouvelle, 1980. 81-167.

Scognamiglio, Concetta Menna. "Il dialogo della bella creanza delle donne in Italia e Francia". Parcours et Rencontres. Mélanges de langue, d' histoire et de littérature français. Ed. Paolo Carile, Giovanni Dotoli et al. Paris: Klincksieck, 1993.471-88.

-Instruction pour les jeunes dames, Par la Mere et Fille d'Alliance (1597). FasanoParigi: Schena-Nizet, 1992.

Scrivano, Riccardo. Cultura e letteratura nel Cinquecento. Roma: Edizioni dell'Ateneo, 1966.

Ughetti, Dante. François d'Amboise (1550-1619). Roma: Bulzoni, 1974.

Valeri, Diego. Conversazioni italiane. Firenze: Olschki, 1968. 